\title{
NNT: Nearest Neighbour Trapezoid Algorithm for IoT WLAN Smart Indoor Localization Leveraging RSSI Height Estimation
}

\author{
Wilford Arigye $\mathbb{D}^{1},{ }^{1}$ Mu Zhou, ${ }^{1}$ Muhammad Junaid Tahir, ${ }^{1}$ Waqas Khalid, ${ }^{1}$ and Qiaolin $\mathbf{P u}^{2}$ \\ ${ }^{1}$ School of Communication and Information Engineering, Chongqing University of Posts and Telecommunications, \\ Chongqing 400065, China \\ ${ }^{2}$ Hong Kong Baptist University, Hong Kong
}

Correspondence should be addressed to Wilford Arigye; 1201910017@stu.cqupt.edu.cn

Received 4 May 2021; Accepted 11 July 2021; Published 2 August 2021

Academic Editor: Abdellah Touhafi

Copyright ( 92021 Wilford Arigye et al. This is an open access article distributed under the Creative Commons Attribution License, which permits unrestricted use, distribution, and reproduction in any medium, provided the original work is properly cited.

Indoor localization as a technique for assisting, or replacing outdoor satellite and cell tower localization systems, has taken a toll in the recent Internet of Things (IoT) era. This IoT drive has prompted increased research towards indoor localization, where fingerprinting, radio mapping as a cost-effective and efficient scheme, is emerging as the best enterprise entrepreneurs choose. However, indoor complex environments comprise of trackable devices (TD) at various heights, such as child trackers, dog tags, TD on the table, TD's in the pockets, and situations such as pedestrians talking on the phone: that is at the height of the ear, amongst others. This paper first investigates and analyses "experimentally" the impact of received signal strength indicator (RSSI) fingerprinting height to construct radio maps for indoor localization. Secondly, it proposes the novel trapezoid path loss model for RSSI estimation and finally the nearest neighbour trapezoid (NNT) algorithm for IoT smart indoor localization leveraging and mitigating the impact of height considered during the offline signal fingerprinting. We further propose approximately 1 meter above the flooring of the target space as the effective fingerprinting height for indoor localization approaches.

\section{Introduction}

Realization of Internet of Things (IoT) technology in recent years for smart cities, smart homes, and integrated government infrastructure services have raised the applicability of location-based services (LBS). Localization fingerprinting in complex $\mathrm{Wi}-\mathrm{Fi}$ indoor environments as a technique to achieve precise indoor positioning has attracted affordable and reliable accuracy ever since the introduction of the RADAR [1,2] and Horus indoor localization systems [3]. Indoor localization usage covers a wide range of aspects, such as emergency response [4], location-based targeted advertising $[5,6]$, indoor robotics navigation [7], and capability that falls short to outdoor satellite navigation systems indoors due to signal fluctuations within complex indoor environments [8]. Indoor positioning technologies such as Bluetooth [9], ultra-wideband [10], radiofrequency technologies [11], microelectromechanical systems [12], wireless local area net- works [13], computer vision [14], magnetic field [15], ultrasonic [16], and infrared signal have been proposed [17].

In this paper, we first experimentally evaluate, test, and analyse the effect of fingerprinting height to indoor localization accuracy using machine learning nearest neighbour in signal space approaches such as nearest neighbour $(\mathrm{NN})$ and $k$-nearest neighbour (KNN) algorithms with various radio maps constructed at different heights. The KNN algorithms return the location estimate as the average of the coordinates of the $k$ neighbours corresponding to the smallest RSS distances to the query RSSI values [2]. Then, we propose and test the performance of the trapezoid path loss model to estimate the RSSI and the trapezoid nearest neighbour algorithm to accurately estimate the mobile location with minimal distance error. Experimental results show better localization accuracy performance by the proposed trapezoid signal distance model than the Euclidean distance deploying the proposed NNT algorithm than the native NN algorithm. 
The contributions of this paper can be summarized as follows:

(1) The proposed system deploys the RSSI-based approach, which requires no additional hardware and easily implemented on/off the shelf mobile devices equipped with the 802.11 family chipsets. Other than relaying of the internal inertia sensors, our proposed technique utilizes the received RSSI to estimate the height of the trackable device

(2) We propose trapezoid signal distance, instead of the Euclidean distance of the received RSSI signal and the fingerprint, as the evaluation function that proves better positioning accuracy

(3) We propose a novel trapezoid model for signal prediction in free space based on proposed trapezoid signal distance other than the log distance model

(4) We propose the nearest neighbour trapezoid algorithm (NNT) for indoor complex fingerprinting localization. Furthermore, we state that the proposed model can be used in any other location system. It provided better and robust localization

Indoor localization systems are discussed in Section 2. Empirical fingerprint construction is presented in Section 3. In Section 4, we describe our proposed trapezoid construction process, the trapezoid path loss model, and the localization algorithm. Section 5 presents the experimental evaluation. Finally, Section 6 concludes this paper.

\section{Related Works}

Indoor localization research has seen a great deal of interest over the decade cutting across various architectures. Several solutions have been proposed by multinational industries and researchers, some requiring dedicated infrastructures such as infrared [18], ultrasound [19], and radiofrequency identification (RFID) [20, 21], thus increasing the cost of deployment. However, RFID emerging techniques to resolve collision detections, such as the enhanced collision detection (ECD) [22], can improve identification rate, time, and slot efficiencies at low cost, whereas some solutions leverage already existing sensor infrastructures, such as Bluetooth [9, 23], frequency modulation (FM) [24], GSM cellular [25], and wireless fidelity (Wi-Fi) signal strengths $[2,3,26,27]$. They deploy techniques such as the angle-of-arrival (AoA) leveraging the angle of incidence of the received signal vectors [28], time-difference-of-arrival (TDOA), the time-offlight (TOF) [29] leveraging the arrival time sequence to measure the delay of the signal, and signal strength fingerprinting. Fingerprint techniques map indoor propagated signals to a specific reference point, without the need to know the transceiver's location and transmit power, as opposed to techniques that rely on building signal propagation models for localization.

WLAN indoor localization system based on fingerprinting comprises of basic two stages. The first stage is radio map construction during the offline surveying stage: During this stage, site survey calibration is carried out to obtain specific reference points at which RSSI sample vectors are sampled at a predetermined height and then saved in the localization server. The second stage is the localization query stage: During this stage, sensor device queried fingerprint vectors at unknown locations are compared to fingerprints in the radio map database in the localization server and then returns the corresponding location estimate that minimizes the mean errors in accordance to the localization algorithms' criterion. Indoor complex environments comprise devices tractable at different heights, such as child trackers, dog tags, mobile devices on the table or in pockets, and pedestrians on phone talking, amongst other height orientations. These diverse height orientations would have an impact on RSSI signal fluctuations; thus, different localization accuracy estimation results during online localization; this is because in most cases offline fingerprints are sampled and constructed at a specific height. This paper, therefore, presents an indepth experimental evaluation to validate this height effect, proposes a novel trapezoid path loss model, and finally proposes novel trapezoid nearest neighbour localization approach.

\section{Fingerprint Localization}

The fingerprint-based localization process consists of offline data collection, radio map construction phase, and online localization estimation phase. Offline construction of the radio map is initialized by the site survey, with grid formation calibrating of the target indoor environment. At each calibrated reference point (RP), we use a prerequisite $\mathrm{Wi}-\mathrm{Fi}$ enabled tractable device (TD) to scan and sample the received signal strength indicator (RSSI) value from hearable transmitter access points (APs) in a predefined time stamp. When a discoverable number of APs are less than 3, the fingerprint signature at that specific RP point is not viable for complex indoor localization environments; thus, the fingerprint surveyor should take note of the AP population within the target environment signal coverage.

Let $N$ be the number of RPs and $L$ be the total number of APs deployed in the signal coverage target floor. We denote the RSSI value form AP $l$ at RP $i$ as $f_{i}^{l}(\mathrm{dBm})$. We sample multiple random fingerprint signals at each predefined RP and then averaged the signal values to find the mean RSSI $\bar{f}_{i}^{l}$ at each RP $i$ from AP $l$ denoted as

$$
\overline{\mathrm{f}}_{i}^{l}=\frac{1}{S_{i}^{l}} \sum_{s=1}^{S_{i}^{l}} f_{i}^{l}(s), i=1, \cdots, N . l=1, \cdots, L
$$

where $f_{i}^{l}(s)$ is the $s^{\text {th }}$ RSSI sample (in $\mathrm{dBm}$ ) at RP $i$ from AP $l$, and $S_{i}^{l}$ is the total number of RSSI samples collected within the predefined time stamp. Then, the fingerprint at RP $i$ is defined as

$$
\mathbf{F}_{i}=\left[\bar{f}_{i}^{1}, \bar{f}_{i}^{2}, \cdots, \bar{f}_{i}^{L}\right]
$$


Forming an interactive radio map matrix as

$$
\Psi=\left(\begin{array}{ccc}
\bar{f}_{1}^{1} & \cdots & \bar{f}_{1}^{L} \\
\vdots & \ddots & \vdots \\
\bar{f}_{N}^{1} & \cdots & \bar{f}_{N}^{L}
\end{array}\right)
$$

Secondly, for each predefined time stamp, we calculate the mean and standard deviation of the RSSI per AP to store in a radio map at the back end of the server. Let $\sigma_{n}^{l}(\mathrm{dBm})$ be the corresponding standard deviation of $S_{n}^{l}$ collected RSSIs.

Similarly, given the online query target measured RSSI $R^{l}$ from AP $\boldsymbol{l}$, the RSSI vector at the target, denoted as $\mathbf{9}$, can be defined as

$$
\vartheta=\left[R^{1}, R^{2}, \cdots, R^{L}\right]
$$

During the data processing, to differentiate the RSSI values within indoor environment, $\mathrm{mW}$, instead of $\mathrm{dBm}$, is used when we consider the random signal level mean, i.e.,

$$
\left.\bar{f}_{i}^{l}\right|_{\mathrm{mW}}=10\left(\left.\bar{f}_{i}^{l}\right|_{\mathrm{dBm}}\right) / 10
$$

which transforms RSSIs from smartphones to values for better signal differentiation. Correspondingly, we also transform RSSI values $t^{l^{\prime}} s$ in $\mathbf{9}$ from $\mathrm{dBm}$ into $\mathrm{mW}$.

\section{NNT Algorithm}

As IoT indoor environments are comprised of localization of persons of interest and transceivers at diverse heights, we propose a novel approach to solve the disparity of the pairwise cluster of the fingerprint RSSI and receiver RSSI by the trapezoidal area between the fingerprint perpendicular heights. Assuming a transceiver Tx at height $H 1$ on $(x 0, y 0$ ) coordinate location in the equal space calibrated indoor space, fingerprint $\mathrm{Fp}$ at height $\mathrm{H} 2$ on $(x 1, y 1)$ coordinate and receiver $R x$ at a height $H 3$ on $(x 2, y 2)$ coordinate, as shown in Figure 1 and the two-dimensional distance relationship in Figure 2.

In order to measure the degree of neighbour's closeness at varying heights, we propose minimizing the proposed trapezoidal distance, which is further achieved by several other minimizations such as the hypotenuse signal distance, the floor distance between the fingerprint and the location device, the differential height between the two. From Figure 1,

$$
\tan Q=\frac{C 1}{(H 1-H 2)}=\frac{C 2}{(H 2-H 3)}=\frac{C 1+C 2}{(H 1-H 3)},
$$

where

$$
C 1=\sqrt{(x 1-x 0)^{2}+(y 1-y 0)^{2}},
$$

$$
h 1=\sqrt{(C 1)^{2}+(H 1-H 2)^{2}} .
$$

4.1. RSSI Distance Model. Natively, during the offline calibration stage, the average RSSI at various fingerprint reference points at different distances $C 1$ from the $T x$ transceiver antenna can be associated with the RSSI distance model as in

$$
\bar{f}_{i}^{l}=10 n \log (\mathrm{C} 1)+\bar{A}_{l}+X_{\sigma}
$$

where $\overline{A_{l}}$ is the average RSSI at a $1 \mathrm{~m}$ distance from $T x$. Assuming a close neighborhood from the Tx antenna nodes in the wireless fidelity network, the transmission indoor space-dependent parameter $n$ remains the same. Thus, $n$ can be determined by

$$
n=\frac{\bar{f}_{i}^{l}-\bar{A}_{l}}{10 \log (C 1)}
$$

During online, the target received RSSI $R^{l}$ of the surrounding transceivers are compared to the fingerprint database RSSI values. Similarly obeying the anticipated RSSI distance model as in

$$
R^{l}=10 n \log (\mathrm{C} 2)+\bar{f}_{i}^{l}
$$

The distance at which the receiver is anticipated is obtained as

$$
\mathrm{C} 2=10\left(\left(R^{l}-\bar{f}_{i}^{l}\right) / 10 n\right) .
$$

Further, we derive the height estimate at which the RSSI is estimated to be recorded as

$$
H 3=\frac{H 2(C 1 /(H 1-H 2))-C 2}{(C 1 /(H 1-H 2))} .
$$

4.2. Trapezoid Path Loss Model. In this section, we propose and define our trapezoid path loss model for indoor radio propagation. Existing models have approximately predicted the RSSI fingerprints, though extremely challenging due to multipath effect and environmental site-specific parameters. From Figure 2, we can derive the following relationship:

$$
Q+90+\alpha=180, \alpha=90-Q
$$

$\sin (90-Q)=\sin 90 \cos Q-\cos 90 \sin Q=\cos Q=\frac{(H 2-H 3)}{h 2}$,

$$
\cos \left(\arctan \left(\frac{C 2}{H 2-H 3}\right)\right)=\frac{1}{\sqrt{1+(C 2 /(H 2-H 3))^{2}}}=\frac{H 2-H 3}{h 2}
$$




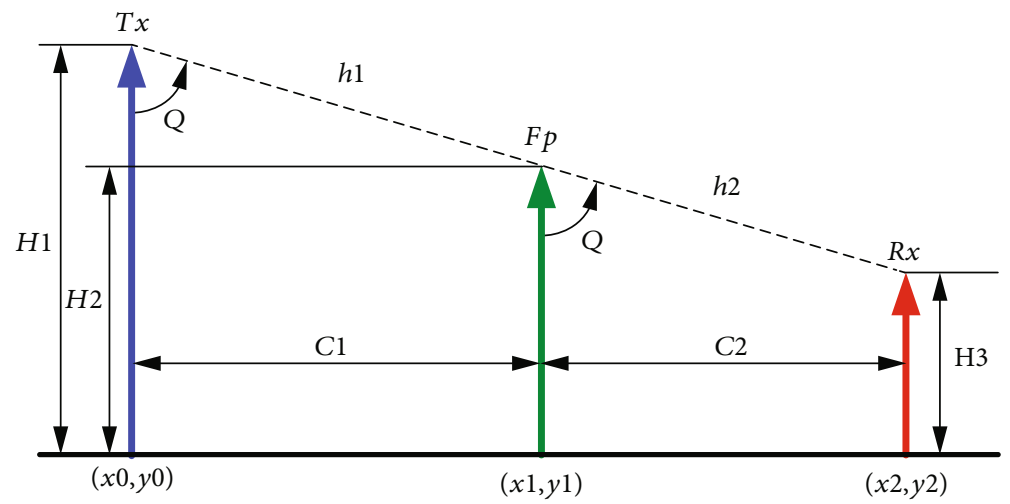

Figure 1: Fingerprinting system perpendicular relationship. The height relationship of the transmitter, fingerprint, and receiver.

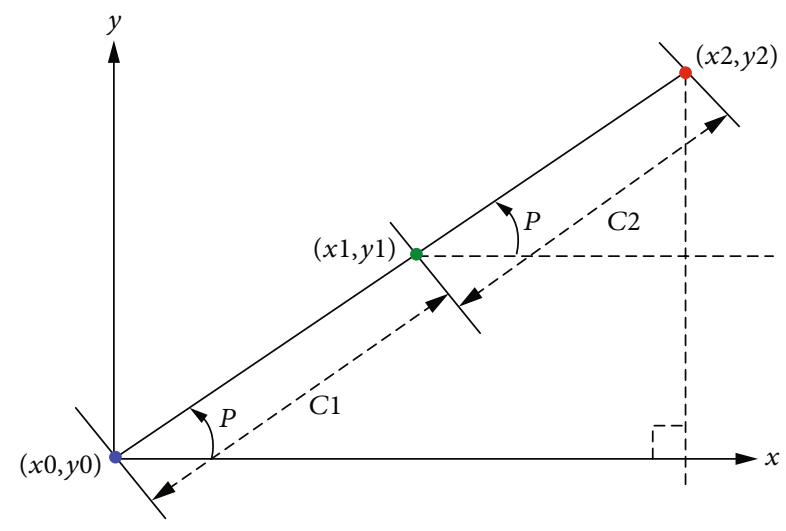

Figure 2: Fingerprinting system floor relationship. The floor distance relationship of the transmitter, the fingerprint, and the receiver.

From Equation (16),

$$
h 2=(H 2-H 3) \times \sqrt{1+\left(\frac{C 2}{H 2-H 3}\right)^{2}} .
$$

The trapezoid nearest neighbour area $A$ is defined as

$$
A=\frac{C 2}{2}(H 2+H 3)=\frac{(H 2+H 3)}{2} \sqrt{(h 2)^{2}-(H 2-H 3)^{2}} .
$$

The proposed trapezoid path loss model that leverages the trapezoid distance can be defined as

$$
R x^{l}=\bar{f}_{i}^{l}+\frac{10 n \log (C 2)}{h 2}
$$

where averaged $R x^{l}$ is the estimated received RSSI, C2 is the estimated distance between the transceiver and receiver, $\bar{f}_{i}^{l}$ is the fingerprint RSSI, and $h 2$ is the proposed trapezoid factor to signal distance that affects the signal between the transceiver and the receiver.
4.3. Proposed Algorithm. Amongst existing main machine learning typical deterministic method for classifying objects based on closest neighbour training examples in the radio map such as $\mathrm{NN}$ and $\mathrm{KNN}$, we propose novel heightspecific NNT and KNNT algorithm for the online localization stage. KNNT aims at returning the position estimate of the current location query as an average of the $k$-neighbours in the radio map resulting from the minimum pairwise trapezoid signal distance $d$, used to query fingerprint in a 2dimensional space.

$$
\widehat{\ell}(\mathbf{R})=\frac{1}{K} \sum_{i=1}^{K} \operatorname{argmin}_{\ell_{i}}\left(d_{i}\right),
$$

where the proposed trapezoid signal distance as in Equation (20) between the reference fingerprint and the observed test fingerprint is calculated as

$$
d_{i}^{2}=h 2_{i}^{l} * \operatorname{sqrt}\left(\sum_{l=1}^{L}\left(\bar{f}_{i}^{l}-R^{l}\right)^{2}\right) .
$$

\section{Experimental Evaluation}

Raw data fingerprinting experiment was carried out on the office floor of our faculty administration building of Chongqing Posts and Telecommunications University (CQUPT), a test bed of about $66 \mathrm{~m}$ wide by $17.1 \mathrm{~m}$ long, whose schematic experimental test floor plan is shown in Figure 3. Several stages include the experimental setup and data acquisition steps in Section 5.1, the initial experiment to determine the effect of height on radio fingerprinting, the proposed trapezoid path loss model, and the proposed trapezoid localization algorithm based on accuracy results and cost of construction.

5.1. Setup and Data Acquisition. In the test bed setup, initial prescans discover various RSSI readings that could be resulting from tethered devices in various offices, which in turn could increase the preprocessing computational cost of the collected data due to increased discoverable TD's. To minimize the processing, we filter out and simplify the process by setting up 5 D-Link APs (DAP 2310) operating at 2.4 GHz IEEE $802.11 \mathrm{~b} / \mathrm{g} / \mathrm{n}$ Wi-Fi standard as baseline testing 


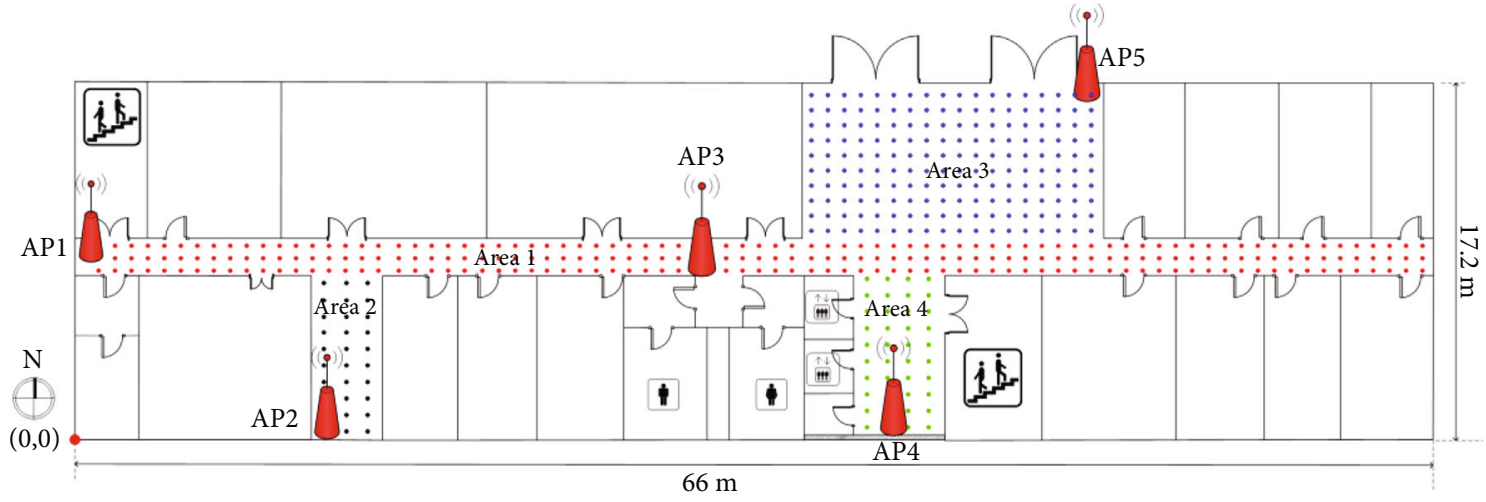

Figure 3: Test bed. Five APs installed on the target floor. 327 RPs calibrated at an interval of $0.8 \mathrm{~m}$.

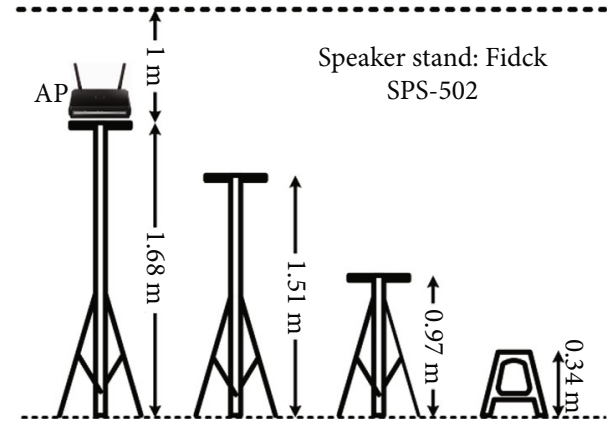

Figure 4: Configuration of the heights.

transceivers. Installed at a height of $1.68 \mathrm{~m}$ on the adjustable Fidck SPs-502 speaker stands, whereas the sampling TDs are at respective heights as described in Figure 4.

During the one-time offline training phase, we calibrate RPs on the floor at respective scaling intervals of $0.8 \mathrm{~m}$ forming a grid. At each grid RP, we sample 120 RSSI values (in $\mathrm{dBm}$ ) as fingerprints within a 1-second time interval using our developed android application facing the northing direction, installed it on a prerequisite Samsung Galaxy GTS7568, and averaged and stored as fingerprints in the localization server, at different heights of $1.51 \mathrm{~m}, 0.97 \mathrm{~m}, 0.34 \mathrm{~m}$, respectively, as illustrated in Figure 4, as well as Figure 5 showing the real image of the various height setups in the test bed. Meanwhile, we further calibrate test RP's, at which respective 120 RSSI values are sampled, averaged, and stored for online unknown locality testing.

Considering the amount of time for the surveyor to interact with the developed APP, our developed android platform sampling APP is simpler and user friendly than in [30], requiring only the reference point name, the interval at which we sample the RSSI. On scan initialization for a predefined time stamp, it records the interval, the RSSI value followed by MAC address of the source transceiver AP. Saving the sampled data into a text file (.txt) format in the security digital (SD) card, from which we later extract RSSI values using MATLAB R2015b running on 64-bit Windows 7 Ultimate desktop equipped with i3 $4160 \mathrm{CPU} 33.60 \mathrm{GHz}$ processor and 4 GB RAM to form an interactive matrix, thus empirical RSS database.
5.2. Height Effect on Localization Accuracy. We experimentally evaluate the localization accuracy of various Wi-Fi fingerprints constructed at different heights for indoors localization to enable use for determining the extent to which the height factor impacts our fingerprint. We use nearest neighbour algorithms of $\mathrm{NN}$ and $\mathrm{KNN}$, where $\mathrm{NN}$ is a special case of the KNN algorithm when the number of neighbours in the localization formulation is equal to one $(k=1)$.

$H 1$ vs. $T 1$ represents radio map fingerprints at height 1 $(H 1=0.34 \mathrm{~m})$ versus testing fingerprints at height 1 $(T 1=0.34 \mathrm{~m})$; this notation is adopted for all the possible height combinations used in the testing (Figure 6), summarized in Table 1. We can see a radio map constructed with fingerprints at heights $H 2$, and test fingerprints at height $T$ 3 , that is "H2 vs. T3" performing better than other comparison fingerprint height combinations. This confirms that when a localization device is at the height of $1.51 \mathrm{~m}$, location fingerprints constructed at $H 2$ result in reduced localization mean error greatly than $H 1$, and $H 3$ fingerprints, respectively, with the same online query test fingerprints at height T3. We further observe that, under different values of the well-known parameter $k$ used by the KNN, the attained localization accuracy differs greatly, with $k=3$ resulting in better performance with combinations of $\mathrm{H} 2$ vs. T3. When the number of neighbour's used during the localization algorithm is equal to $1(k=1)$, resulting results are attributed to the $\mathrm{NN}$ algorithm. From this point, we select and proposed $\mathrm{H} 2$ as our fingerprinting height for the analysis of the proposed trapezoid path loss model and finally the proposed localization technique.

5.3. Trapezoid Pass Loss Model Analysis. Efficient, applicable RSSI signal prediction is a key to indoor localization in the era of IoT, however, due to the diversity of the height of location-based devices, accurate signal modelling at different receiver heights on the same location possesses a challenge due to multipath effect, refraction, diffraction, and reflection of the signal by the complex indoor environments. Considering the lobby area (Area 3), we perform a comparison to the choices of minimization factors from the following enabling features such as the proposed trapezoid signal distance (blue colour), the signal distance (red colour), the floor distance (green colour), and the trapezoid area (black colour). From 


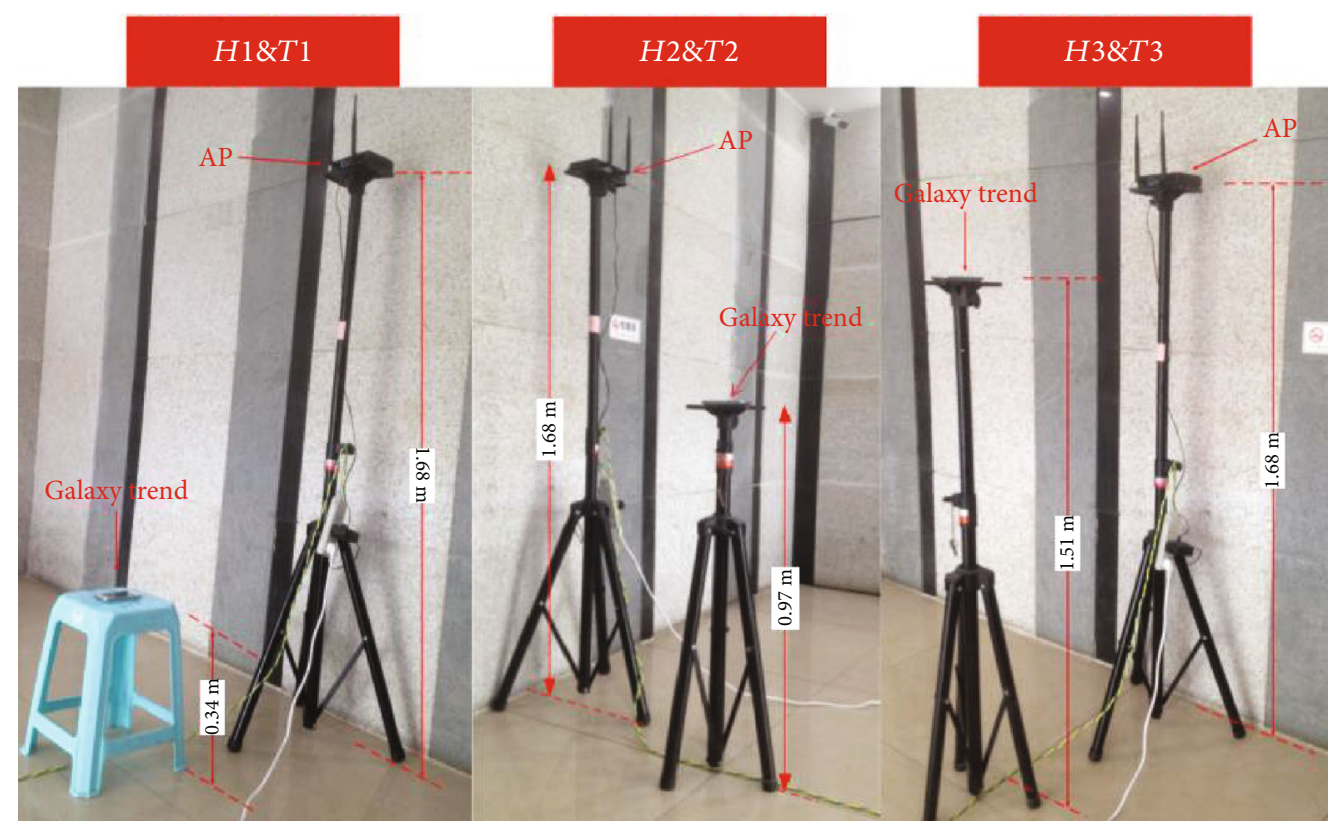

Figure 5: Configuration of the heights picture. $H 1$ vs. $T 1, H 2$ vs. $T 2$, and $H 3$ vs. $T 3$.

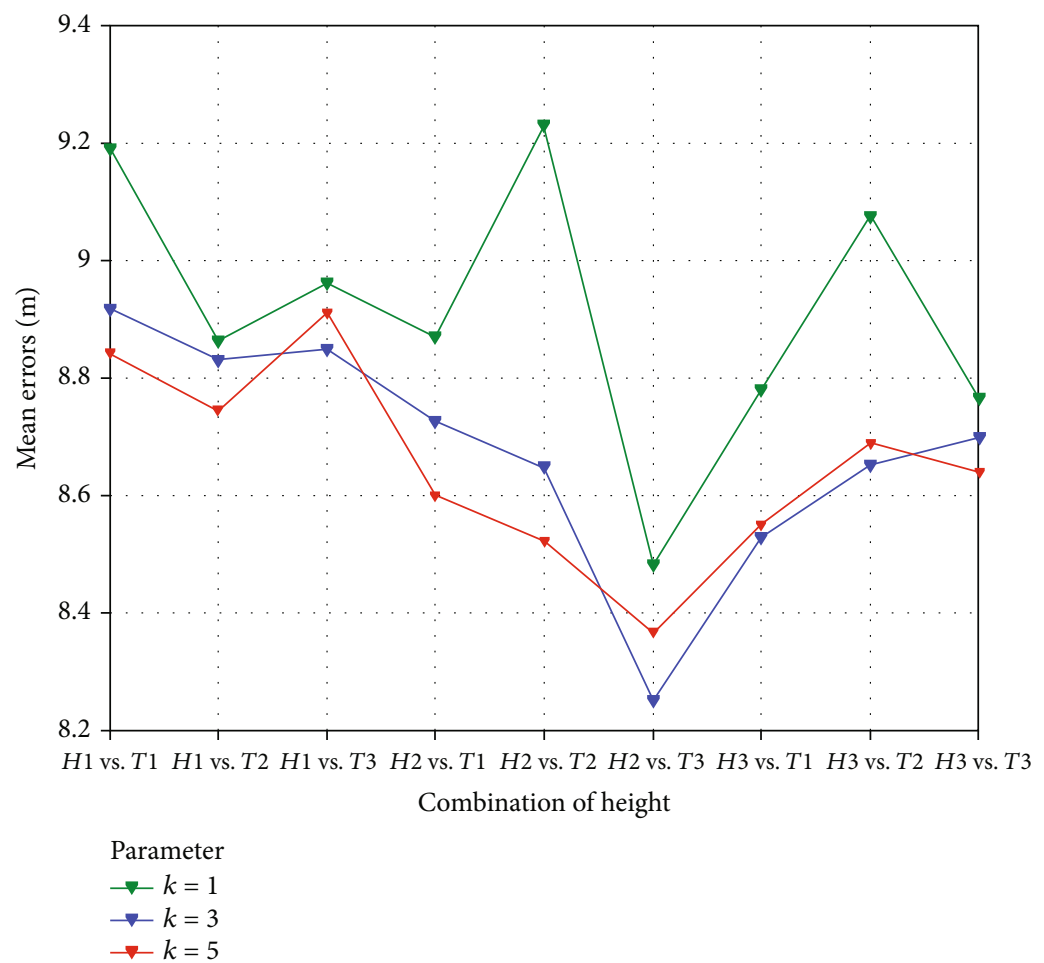

FIGURE 6: Comparison of location mean errors under difference combinations of heights and $k$ parameter values.

TABLE 1: Summary of height combinations.

\begin{tabular}{llll}
\hline Height & $0.34(\mathrm{~m})$ & $0.97(\mathrm{~m})$ & $1.51(\mathrm{~m})$ \\
\hline $0.34(\mathrm{~m})$ & $H 1$ vs. $T 1$ & $H 1$ vs. T2 & $H 1$ vs. T3 \\
$0.97(\mathrm{~m})$ & $H 2$ vs. $T 1$ & $H 2$ vs. T2 & $H 2$ vs. T3 \\
$1.51(\mathrm{~m})$ & $H 3$ vs. $T 1$ & $H 3$ vs. T2 & $H 3$ vs. T3 \\
\hline
\end{tabular}

the comparison, we observe overall superior localization accuracy performance of the proposed prediction path loss model leveraging height estimation than peers, as seen in Figure 7. Furthermore, analysing the localization accuracy by the NNT algorithm that leverages trapezoid path loss model at each TP location in Area 3, as seen in Figure 8. We observe robustness with NNT localization mean errors of range 0 meters (floor) such as TP location 6, TP location 


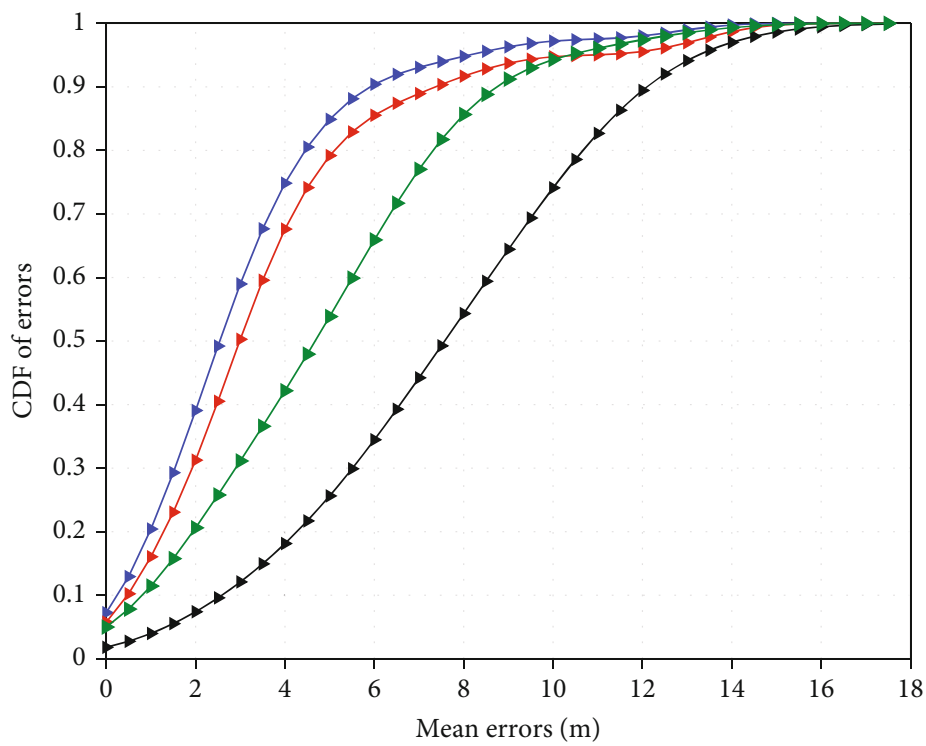

Deterministic factors

$\rightarrow$ Proposed distance

$\rightarrow$ Trapezoid area

$\rightarrow$ Euclidean distance

$\rightarrow$ Floor distance

FIGURE 7: CDF comparison of deterministic factors.

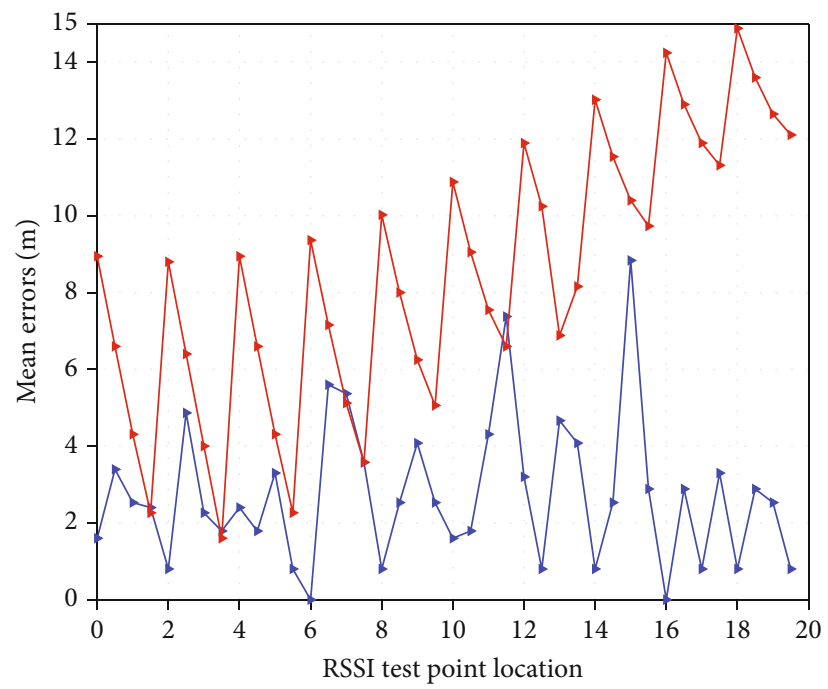

Path loss model

$\rightarrow$ Trapezoid

$\rightarrow$ Log distance

FIgURE 8: Comparison of location accuracy by path loss models. Area 3 location mean errors at 40 test TPs. 


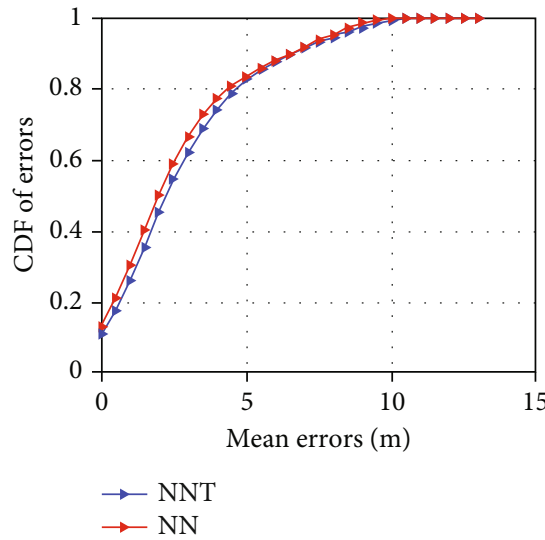

(a)

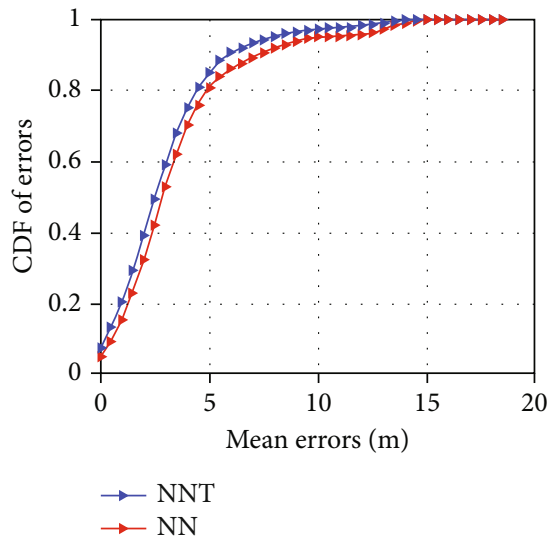

(c)

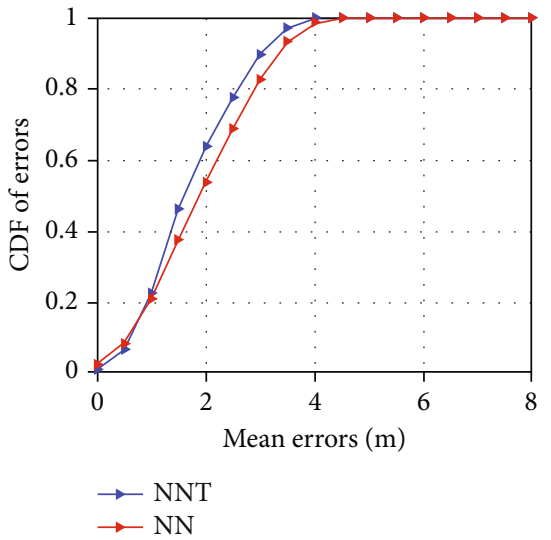

(b)

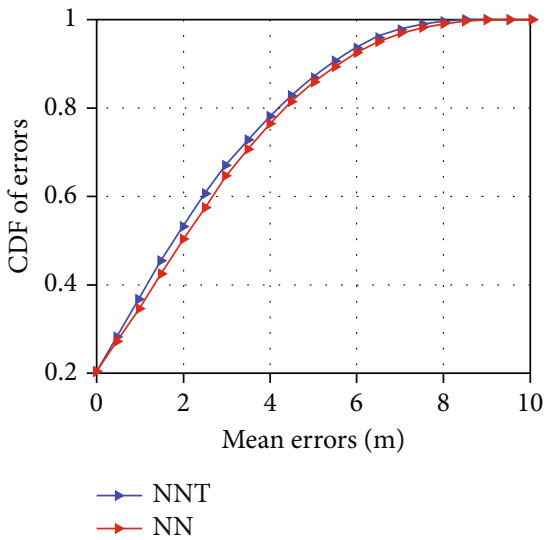

(d)

FIgURE 9: Localization sub area accuracy. (a) Area 1 corridor with LoS AP1 and AP3. (b) Area 2 left lower space with LoS AP2. (c) Area 3 middle lobby with LoS AP5. (d) Area 4 lower middle lobby with LoS AP4.

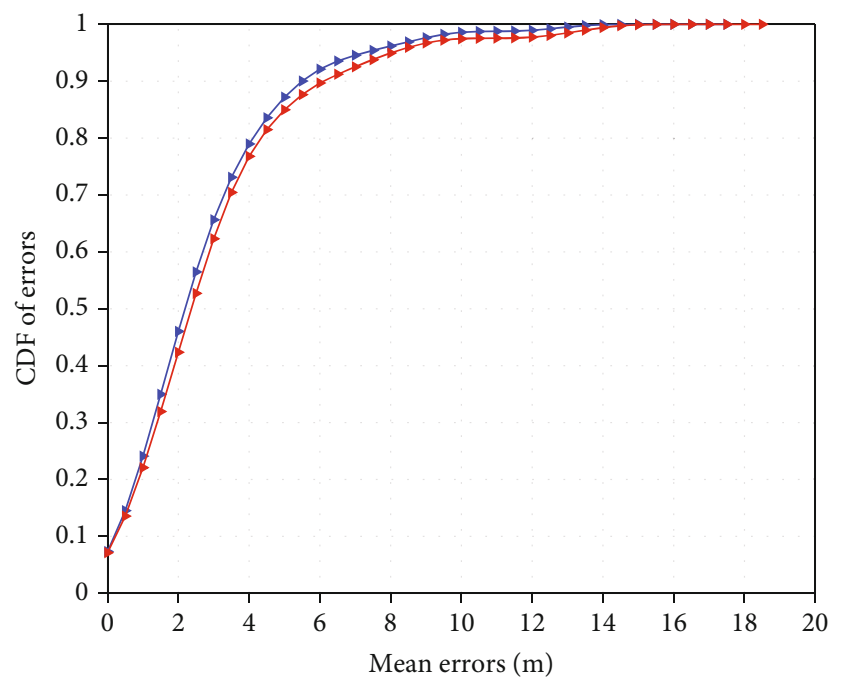

Algorithm

$\rightarrow$ NNT

$\rightarrow \mathrm{NN}$

FIGURE 10: Total floor. Localization mean errors in meter within a complex environment, total floor coverage.
16 and 9 meters (ceiling), with a median of 2 meters compared to NN range of 2 meters (floor) to 15 meters (ceiling) with all RSSI test RP's.

5.4. Localization Accuracy Analysis. We evaluate the accuracy of the proposed NNT localization algorithm by computation of the mean error with the well-known NN algorithm in different environments, as seen from the Figure 9, below for each areas subsection, and the general total floor, we observe the NNT algorithm (blue) outperforming the native NN algorithm (red), both in room base localization and total floor base localization Figure 10.

\section{Conclusions}

As the demand increases for indoor LBSs in the IoT era, WiFi-based fingerprinting as a key low-cost approach to precise indoor navigation and positioning keeps increasing. This paper firstly presents an extensive experimental analysis on the effect of the height, chosen by the offline site surveyor for sampling RSSI data in one-sided heading, as a minimum measure during the fingerprint radio map construction. We further note that pedestrians and traceable valuables indoors can be found at diverse heights; thus, the same could 
reversibly affect the localization radio map, directly impacting the localization accuracy. From this evaluation, we observe the effect of AP's installation heights versus the localization height in complex environments and further propose that radiofrequency-based indoor fingerprinting to be sampled at approximately $1 \mathrm{~m}$ above the floor of localization interest.

Secondly, we propose a novel trapezoid path loss model to better estimate indoor RSSI fingerprint characteristics due to changing height. On the same basis, we finally propose a novel trapezoid-based nearest neighbour indoor localization scheme that leverages online RSSI to dynamically predict and update the RSSI in real time. Comparing with the classic nearest neighbour algorithm for localization accuracy performance, the experimental findings clearly show that the proposed algorithms can better predict RSSI with dynamic indoor coverage and improve the positioning accuracy.

\section{Data Availability}

The data is available on request.

\section{Conflicts of Interest}

The authors declare that there is no conflict of interest regarding the publication of this paper.

\section{Acknowledgments}

This work was supported in part by the Science and Technology Research Program of Chongqing Municipal Education Commission (KJZD-K202000605, KJQN202000630), the Chongqing Natural Science Foundation Project (cstc2020jcyj-msxmX0842), the National Natural Science Foundation of China (61771083, 61771209), and the Program for Changjiang Scholars and Innovative Research Team in the University (IRT1299).

\section{References}

[1] D. Macagnamo, G. Destino, and G. Abreu, "Indoor positioning: a key enabling technology for IoT applications," in 2014 IEEE World Forum on Internet of Things (WF-IoT), pp. 117118, Seoul, Korea, 2014.

[2] P. Bahl and V. N. Padmanabhan, "RADAR: an in-building RFbased user location and tracking system," in Proceedings IEEE INFOCOM 2000. Conference on Computer Communications. Nineteenth Annual Joint Conference of the IEEE Computer and Communications Societies (Cat. No.00CH37064), Tel Aviv, Israel, 2000.

[3] M. Youssef and A. Agrawala, "The Horus WLAN location determination system," in Proceedings of the 3rd international conference on Mobile systems, applications, and services MobiSys '05, pp. 205-218, ACM: New York, 2005.

[4] E. D. Manley and J. Deogun, "Location learning for smart homes," in 21st International Conference on Advanced Information Networking and Applications Workshops (AINAW'07),, Niagara Falls, ON, Canada, May 2007.

[5] P. Bellavista, A. K, and S. Helal, "Location-based services: back to the future," IEEE Pervasive Computing, vol. 7, no. 2, pp. 8589, 2008.
[6] L. Aolto, N. Gothlin, J. Korhonen, and T. Ojala, "Bluetooth and WAP push based location-aware mobile advertising system," in Proceedings of the 2nd International Conference on Mobile Systems, Applications, and Services, ser. MobiSys '04, pp. 49-58, New York, NY, USA: ACM, 2004.

[7] B. Sohn, J. Lee, H. Chae, and W. Yu, "Localization system for mobile robot using wireless communication with IR landmark," in The Proceedings of First International Conference on Robot Communication and Coordination, Piscataway, NJ, USA: IEEE Press, 2007.

[8] P. Dana, Global positioning system overview, Department of Geography university of Colorado, 1999.

[9] P. Davidson and R. Piche, "A survey of selected indoor positioning methods for smartphones," IEEE Communications Surveys \& Tutorials, vol. 19, no. 2, pp. 1347-1370, 2017.

[10] A. Ren, F. Zhou, A. Rahman, X. Wang, N. Zhao, and X. Yang, "A study of indoor positioning based on UWB base-station configurations," in 2017 IEEE 2nd Advanced Information Technology, Electronic and Automation Control Conference (IAEAC), pp. 1939-1943, Chongqing, China, March 2017.

[11] S. S. Saab and Z. S. Nakad, "A standalone RFID indoor positioning system using passive tags," IEEE Transactions on Industrial Electronics, vol. 58, no. 5, pp. 1961-1970, 2011.

[12] G. Li, E. Geng, Z. Ye, Y. Xu, J. Lin, and Y. Pang, "Indoor positioning algorithm based on the improved RSSI distance model," Sensors, vol. 18, no. 9, p. 2820, 2018.

[13] C. Wu, Q. Mu, Z. Zhang, Y. Jin, Z. Wang, and G. Shi, "Indoor positioning system based on inertial MEMS sensors: design and realization," in 2016 IEEE International Conference on Cyber Technology in Automation, Control, and Intelligent Systems (CYBER),, pp. 370-375, Chengdu, China, June 2016.

[14] Y. Tian, D. Shigaki, W. Wang, and C.-J. Ahn, “A weighted least-squares method using received signal strength measurements for WLAN indoor positioning system," in 2017 20th International Symposium on Wireless Personal Multimedia Communications (WPMC),, pp. 310-314, Bali, Indonesia, December 2017.

[15] J. Kim and H. Jun, "Vision-based location positioning using augmented reality for indoor navigation," IEEE Transactions on Consumer Electronics, vol. 54, no. 3, pp. 954-962, 2008.

[16] V. Pasku, A. de Angelis, M. Dionigi, G. de Angelis, A. Moschitta, and P. Carbone, "A positioning system based on low-frequency magnetic fields," IEEE Transactions on Industrial Electronics, vol. 63, no. 4, pp. 2457-2468, 2016.

[17] J. Qi and G. P. Liu, “A robust high-accuracy ultrasound indoor positioning system based on a wireless sensor network," Sensors, vol. 17, no. 11, p. 2554, 2017.

[18] T. Raharijaona, R. Mawonou, T. V. Nguyen et al., "Local positioning system using flickering infrared LEDs," Sensors, vol. 17, no. 11, p. 2518, 2017.

[19] N. B. Priyantha, A. Chakraborty, and H. Balakrishman, "The Cricket location-support system," in Proceedings of the 6th annual international conference on Mobile computing and networking - MobiCom '00, pp. 32-43, New York, NY, USA: ACM, 2000.

[20] D. Hahnel, W. Burgard, D. Fox, K. Fishkin, and M. Philipose, "Mapping and localization with RFID technology," in IEEE International Conference on Robotics and Automation, 2004. Proceedings. ICRA '04. 2004, New Orleans, LA, USA, April 2004.

[21] L. M. Ni, Y. Liu, Y. C. Lau, and A. P. Patil, "LANDMARC: indoor location sensing using active RFID," in Proceedings of 
the First IEEE International Conference on Pervasive Computing and Communications, 2003. (PerCom 2003),, pp. 407-415, Fort Worth, TX, USA, 2003.

[22] J. Su, R. Xu, S. Yu, B. Wang, and J. Wang, "Idle slots skipped mechanism based tag identification algorithm with enhanced collision detection," KSII Transactions on Internet and Information Systems, vol. 14, no. 5, pp. 2294-2309, 2020.

[23] M. Hossain and W. S. Soh, "A comprehensive study of Bluetooth signal for localization," in 2007 IEEE 18th International Symposium on Personal, Indoor and Mobile Radio Communications, Athens, Greece, Sept 2007.

[24] Y. Chen, D. Lymberopoulos, J. Liu, and B. Priyantha, "FMbased indoor localization," in Proceedings of the 10th international conference on Mobile systems, applications, and services - MobiSys '12, pp. 169-182, ACM: New York, 2012.

[25] H. Buyruk, A. K. Keskin, S. Sendil et al., "RF fingerprinting based GSM indoor localization," in 2013 21st Signal Processing and Communications Applications Conference (SIU), Haspolat, Turkey, 2013.

[26] A. Goswami, L. E. Ortiz, and S. R. Das, "WiGEM," in Proceedings of the Seventh COnference on emerging Networking EXperiments and Technologies on - CoNEXT '11, New York, NY, USA: ACM, 2011,.

[27] E. Martin, O. Vinyals, G. Friedland, and R. Bajcsy, "Precise indoor localization using smart phones," in Proceedings of the international conference on Multimedia - MM '10, pp. 787-790, New York, NY, USA: ACM, 2010.

[28] S. Sen, J. Lee, K.-H. Kim, and P. Congdon, "Avoiding multipath to revive inbuilding WiFi localization," in Proceeding of the 11th annual international conference on Mobile systems, applications, and services - MobiSys '13, ACM: New York, 2013.

[29] S. Lanzisera, D. Zats, and K. S. J. Pister, "Radio frequency timeof-flight distance measurement for low-cost wireless sensor locazation," vol. 11, Tech. Rep. 3, IEEE Sensors Journal, 2011.

[30] D. Mascharka and E. Manley, "LIPS: learning based indoor positioning system using mobile phone sensors," in 2016 13th IEEE Annual Consumer Communications \& Networking Conference (CCNC), pp. 968-971, Las Vegas, NV, USA, 2016. 